\title{
Single Wall Closed-form Differential Ultrasound Calibration
}

\author{
Mohammad Najafi, Narges Afsham, Purang Abolmaesumi, Robert Rohling \\ Department of Electrical and Computer Engineering, University of British Columbia, Vancouver, \\ BC, Canada
}

\begin{abstract}
In freehand 3D ultrasound, images are acquired while the position of the transducer is recorded with a tracking device. Calibration is essential in this technique to find the transformation from the image coordinates to the reference coordinate system. The single wall technique is a common calibration method because a simple plane phantom is used. Despite its advantages, such as ease of phantom construction and image analysis, this method requires large number of images to converge to the solution. One reason is a lack of a closed-form solution. Also, the technique uses slightly illconditioned sets of equations with a high condition number due to limited range of scanning motions that produce clear images of the plane. Here, a novel closed-form formulation has been proposed for the single wall calibration technique. Also, differential measurements of the plane image are used instead of absolute plane detection to improve accuracy. The closed-form solution leads to more accurate and robust results while providing an insight into understanding error propagation and finding the optimal set of transducer poses. Results have been compared to the conventional single wall technique. A residual error of $0.14 \mathrm{~mm}$ is achieved for the proposed method compared to $0.91 \mathrm{~mm}$ in the conventional approach.
\end{abstract}

Keywords: Free-hand ultrasound, calibration, single wall phantom, closed-form, differential.

\section{INTRODUCTION}

In freehand 3D ultrasound, which is a technique of acquiring ultrasound images with a tracked transducer, one important step is transducer calibration. It provides the transformation that relates any pixel in the ultrasound image to the corresponding point in a 3D world coordinate system.

A variety of approaches for calibration of ultrasound have been investigated and they have been compared in terms of reconstruction accuracy, reproducibility, and acquisition time ${ }^{1}$. One typical method of calibration is to image an artificial object, known as a phantom, with known geometrical parameters, and combine the prior knowledge of the phantom with its ultrasound images to solve for the calibration parameters. Image features of the phantom can be points, lines or more complex shapes.

Point-based methods are performed by repetitive scanning of a point target such as a spherical fiducial, intersection of two wires, or the tip of a stylus ${ }^{2}$. Since aligning the ultrasound beam at a single point is not easy, scanning along strings or wires can be used instead of single location. The three-wire phantom ${ }^{1}$, Hopkins phantom ${ }^{3}$, and $\mathrm{Z}$ or $\mathrm{N}$ shaped phantoms ${ }^{4}$, are examples of these methods. The final accuracy of point-based or wire-based methods are highly dependent on the accuracy of manual or semi-automatic selection of the point or line centroid in ultrasound images ${ }^{1}$. Given the presence of noise and artifacts, it is argued that a line is segmented easier and more accurately than a point ${ }^{1}$.

The single wall phantom and its enhanced version, the Cambridge phantom ${ }^{5}$, take advantage of this idea and facilitate the automatic segmentation of the line. The Cambridge phantom is very accurate but requires custom apparatus, accurate mounting of the probe, and is difficult to use for a novice user ${ }^{1}$. The single wall phantom is simply a plate, made of e.g. plexiglass, and does not require construction of a phantom but is less accurate especially for low numbers of acquired images acquired approximately perpendicular to the plate (when the plate appears as a clear sharp line). Here it is worth mentioning that wedge phantoms ${ }^{6,7}$ also use planar surfaces but they require accurate and specific phantom design in comparison with the simple design of the wall phantom.

One idea to improve the accuracy of the calibration is to use differential measurements instead of absolute position of the features in the image. In fact, in the single wall technique, the slope of the line is a differential measurement whose accuracy is less dependent on the sharpness of the line. The solution of the conventional single wall technique ${ }^{5}$ is based

Medical Imaging 2012: Image-Guided Procedures, Robotic Interventions, and Modeling,

edited by David R. Holmes III, Kenneth H. Wong, Proc. of SPIE Vol. 8316, 83162A

(C) 2012 SPIE · CCC code: 1605-7422/12/\$18 - doi: 10.1117/12.911459

Proc. of SPIE Vol. 8316 83162A-1 
on measuring the absolute position of points along the line in the image and solves for the calibration parameters by minimizing the residual error. The method is iterative due to lack of a closed-form solution. It also has slightly illconditioned sets of equations due to the limited range of scanning motions that produce clear images ${ }^{5}$.

Here, a closed-form method is proposed that first solves for the rotation components of the calibration matrix using the differential measurements (line slopes) and then, solves for the translation components. This improves the accuracy, and at the same time provides an insight into finding the optimal set of poses for the transducer.

\section{METHODS}

\subsection{Mathematical Framework}

The calibration goal is to find the six degree-of-freedom transformation from the image to the transducer coordinate system $\left({ }^{T} T_{I}\right)$. The transformation from the transducer to the reference coordinate system $\left({ }^{R} T_{T}\right)$ is known by the readings from the tracker (Figure 1).

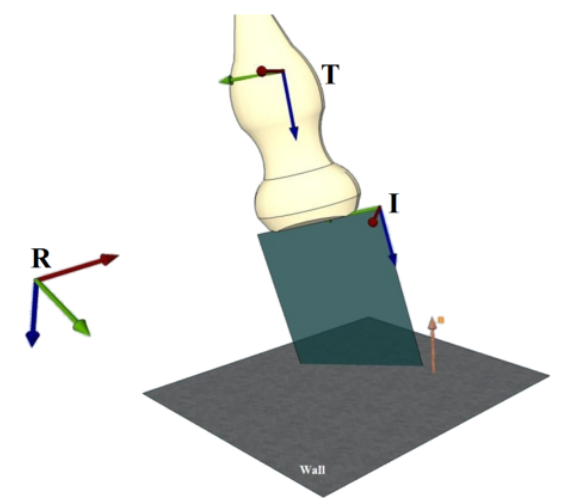

Figure 1. Showing coordinates systems of the Tracker (reference), transducer (T) and the ultrasound image (I) in the calibration setup.

The method is based on scanning a surface with the tracked ultrasound transducer in several different poses. The rotation part of the ${ }^{T} T_{I}$ transformation $\left(R_{x}\right)$ is first calculated using slopes of the lines in the images. The transformation from the image to the reference, ${ }^{R} T_{I}$, can be defined from two free vectors $\vec{U}_{i}$ and $\vec{V}_{i}$ and a point $P_{0_{i}}$ as follows. $\vec{U}_{i}$ is a unit vector in the direction passing through the center of array elements (lateral) and $\vec{V}_{i}$ is a unit vector in the direction of ultrasound beam (axial). $\quad P_{0_{i}}$ is the origin of imaging plane in the reference coordinate system, which is the same as the translation vector in ${ }^{R} T_{I}$. Pixel to millimeter ratios in axial and lateral direction are defined as $S_{x}$ and $S_{y}$, respectively. Here, it has been assumed that $S_{x}$ is known from the transducer specifications provided by the manufacturer, but $S_{y}$ is unknown and is solved in the algorithm.

Using the above notations for each pose of the transducer we have:

$$
\vec{U}_{i}={ }^{R} R_{T}{ }^{T} R_{I}\left[\begin{array}{l}
1 \\
0 \\
0
\end{array}\right], \vec{V}_{i}={ }^{R} R_{T}{ }^{T} R_{I}\left[\begin{array}{l}
0 \\
1 \\
0
\end{array}\right] .
$$


Here ${ }^{T} R_{I}\left(=R_{x}\right)$ is the rotation part of the ${ }^{T} T_{I}$ transform. And ${ }^{R} R_{T}\left(=R_{i}\right)$ is the rotation part of the transducer to reference transform, ${ }^{R} T_{T}$, which is known and comes from the tracker for each pose (i). Considering the value of $\vec{U}_{i}$ and $\vec{V}_{i}$ at the initial pose of the transducer $\left(\vec{U}_{0}\right.$ and $\left.\vec{V}_{0}\right)$, we can define them at pose $i$ using Eq. 1 .

$$
\vec{U}_{i}=R_{i}\left(R_{0}\right)^{-1} \vec{U}_{0}=R_{i}^{d} \vec{U}_{0}, \vec{V}_{i}=R_{i}\left(R_{0}\right)^{-1} \vec{V}_{0}=R_{i}^{d} \vec{V}_{0}
$$

$R_{i}^{d}$ is the relative rotation from the initial pose to the $i^{\text {th }}$ pose, which is known since both $R_{0}$ and $R_{i}$ are determined from the tracker.

Also the origin of the image $\left(P_{0_{i}}\right)$ can be defined as:

$$
P_{0_{i}}={ }^{R} T_{T}{ }^{T} T_{I}\left[\begin{array}{l}
0 \\
0 \\
0 \\
1
\end{array}\right]={ }^{I} R_{T}{ }^{T} \vec{t}_{I}+{ }^{R} t_{T}=R_{i} \vec{t}+\vec{t}_{i},
$$

where $\vec{t}$ is the unknown translation and $\vec{t}_{i}$ is the transducer to reference translation. Therefore each pixel of the image $(\mathrm{x}, \mathrm{y})$ can be described in the reference coordinates as:

$$
P=P_{0 i}+S_{x} x \vec{U}_{i}+S_{y} y \vec{V}_{i}
$$

\subsection{Closed-form Solution}

Considering the pixels on the line appeared in the ultrasound image from the intersection of the wall and the ultrasound image plane, they should satisfy the plane equation (Figure 2):

$$
\left[\left(P_{0 i}+S_{x} x_{1} \vec{U}_{i}+S_{y} y_{1} \vec{V}_{i}\right)-Q\right] \cdot \vec{n}=0
$$

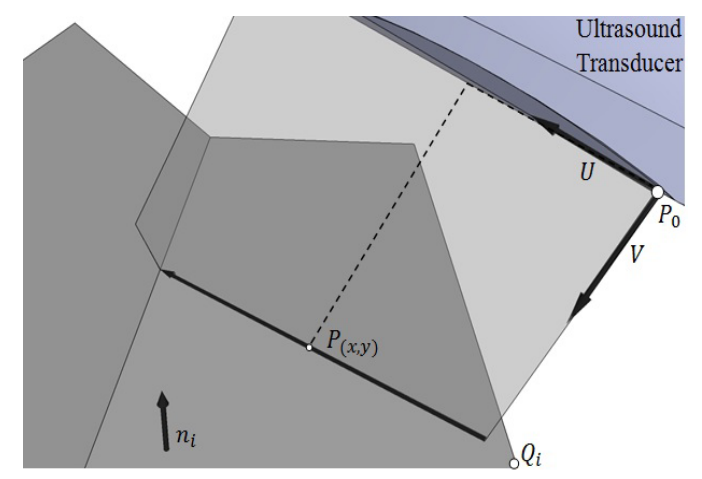

Figure 2. A line will appear in the ultrasound image from the intersection of the wall. Any pixel on the line $\mathrm{P}_{(\mathrm{x}, \mathrm{y})}$ should satisfy the plane equation.

Assume another point of image on the line $\left(\mathrm{x}_{2}, \mathrm{y}_{2}\right)$; by subtracting Eq. 5 for the two points and then dividing by $S_{x}$, and then dividing by $\Delta y$ we have:.

$$
\Delta x \vec{U}_{i} \cdot \vec{n}+k \Delta y \vec{V}_{i} \cdot \vec{n}=0 \Rightarrow \vec{U}_{i} \cdot \vec{n}+k m \vec{V}_{i} \cdot \vec{n}=0,
$$


where $k=\frac{S_{y}}{S_{x}}, \Delta x=x_{2}-x_{1}, m=\frac{\Delta y}{\Delta x}$ and $\Delta y=y_{2}-y_{1}$.

Now by substituting Eq. 2 into Eq. 6:

$$
R_{i}^{d} \vec{U}_{0} \cdot \vec{n}+k m_{i} R_{i}^{d} \vec{V}_{0} \cdot \vec{n}=0
$$

In the above equation $\vec{U}_{03 \times 1}, \vec{V}_{03 \times 1}$ and $\vec{n}_{3 \times 1}$ are unknown and $m_{i}$ and $R_{i}^{d}{ }_{3 \times 3}$ are known. To solve this equation now we use this property of the Kronecker product:

$$
\operatorname{vec}(A X B)=\left(B^{t} \otimes A\right) \operatorname{vec}(X) .
$$

The above equation means that taking vec from the product of three matrixes can be written as the Kronecker product of the last and the first multiplied by the vec of the middle matrix.

Here by taking vec from two sides of Eq. 7 we have:

$$
\left(\vec{U}_{0}^{t} \otimes \vec{n}^{t}\right) \operatorname{vec}\left(R_{i}^{d}\right)+k m_{i}\left(\vec{V}_{0}^{t} \otimes \vec{n}^{t}\right) \operatorname{vec}\left(R_{i}^{d}\right)=0 .
$$

Now by putting the above linear equation for all poses $(i=1$ to $N)$ into matrix form, we have:

$$
\left[\begin{array}{cccccc}
R_{1_{11}}^{d} & \cdots & R_{1_{33}}^{d} & m_{1} R_{1_{11}}^{d} & \cdots & m_{1} R_{1_{33}}^{d} \\
\vdots & \vdots & \ddots & \vdots & \ddots & \vdots \\
R_{N_{11}}^{d} & \cdots & R_{N_{33}}^{d} & m_{N} R_{N_{11}}^{d} & \cdots & m_{N} R_{N_{33}}^{d}
\end{array}\right]_{N \times 18}\left[\begin{array}{c}
x_{1} \\
\vdots \\
x_{9} \\
k y_{1} \\
\vdots \\
k y_{9}
\end{array}\right]_{18 \times 1}=[0]_{N \times 1},
$$

where $\vec{x}_{9 \times 1}=\left(\vec{U}_{0}^{t} \otimes \vec{n}^{t}\right)^{t}$ and $\vec{y}_{9 \times 1}=\left(\vec{V}_{0}^{t} \otimes \vec{n}^{t}\right)^{t}$.

The goal is to find $X=\left[\begin{array}{llllll}x_{1} & \cdots & x_{9} & k y_{1} & \cdots & k y_{9}\end{array}\right]$, the null space of the left side matrix and the answer should be scaled to satisfy:

$$
\|X(1: 9)\|=\left\|\vec{x}_{9 \times 1}\right\|=1
$$

which is obvious since $\vec{U}_{0}, \vec{V}_{0}$ and $\vec{n}$ are unit vectors. To find $R_{x}$ we can use Eq. 1 for any pose (i) by substituting calculated values of $\vec{U}_{0}$ and $\vec{V}_{0}$ from the above solution.

We can easily find $k$ using the below equation:

$$
\|X(10: 18)\|=k\left\|\vec{y}_{9 \times 1}\right\|=k .
$$

So far we have solved the rotation part $\left(R_{x}\right)$ of the calibration matrix $\left({ }^{T} T_{I}\right)$. To solve the translation part $(\vec{t})$ we substitute Eq. 3 into Eq. 5:

$$
\left(R_{i} \vec{t}+\vec{t}_{i}\right) \cdot \vec{n}+S_{x} x_{i} \vec{U}_{i} \cdot \vec{n}+S_{y} y_{i} \vec{V}_{i} \cdot \vec{n}=Q^{t} \cdot \vec{n}=d,
$$

where $d$ is a parameter of the plane equation which is also unknown. 
Eq. 13 is a linear equation in which all the parameters except $\vec{t}$ and $d$ are known. Now by considering Eq. 14 as:

$$
c_{i}=S_{x} x_{i} \vec{U}_{i} \cdot \vec{n}+S_{y} y_{i} \vec{V}_{i} \cdot \vec{n}, \quad \vec{n}_{i}=\vec{n}^{t} R_{i}, \quad G=\left[\begin{array}{c}
{\left[n_{1}{ }^{t},-1\right]} \\
\vdots \\
{\left[n_{N}{ }^{t},-1\right]}
\end{array}\right] .
$$

Then we can rewrite Eq. 13 as:

$$
G\left[\begin{array}{l}
\vec{t} \\
d
\end{array}\right]=-\left[\begin{array}{c}
c_{1} \\
\vdots \\
c_{N}
\end{array}\right]
$$

Eq. 15 can now be easily solved in a least-squares sense to find $\vec{t}$ and $d$ :

$$
\left[\begin{array}{l}
\vec{t} \\
d
\end{array}\right]=-\left(G^{t} G\right)^{-1} G^{t}\left[\begin{array}{c}
c_{1} \\
\vdots \\
c_{N}
\end{array}\right]
$$

\section{EXPERIMENTS AND RESULTS}

The calibration experimental setup consists of SonixTouch ultrasound machine (Ultrasonix Medical Corporation, Richmond, BC, Canada), L14-5 $10 \mathrm{MHz}$ linear 2D ultrasound transducer, Optotrak Certus optical tracker (Northern Digital Inc, Waterloo, Ontario, Canada) and an aluminum plate immersed in a water tank. The tracker identifies the pose of the ultrasound transducer by locating the infrared markers attached to the transducer. Ultrasound images of the aluminum plate (wall) for 40 different poses of the transducer along with the pose of the transducer have been recorded. A combination of rotational and translational movements has been applied to the transducer to generate the poses as suggested in ${ }^{5}$.

The automatic line detection algorithm in ${ }^{5}$ has been implemented to find the lines in the images. Using the line slopes and the described method, the rotation part of the calibration transform is first calculated. To make the solution more robust, the unity constraints of $\vec{U}_{0}, \vec{V}_{0}$ and $\vec{n}$ have been considered as regularization terms while solving the set of linear equations in Eq. (10) with an iterative solver. The condition number is less than 1000; indicating good conditioning. To solve the translation part using Eq. (14), first the values of $c_{i}$ are found from calculated $\vec{U}_{i}, \vec{V}_{i}$ and $\vec{n}$ from the previous part and using the measured y-intercept of the lines. The solution of Eq. (14) gives the translation part $(\vec{t})$ and the wall to origin distance $(d)$. The accuracy of the proposed method has been compared to the conventional single wall technique using the same data set from the line extraction software. As suggested $\mathrm{in}^{5}$, the equations are solved using the Levenberg-Marquardt method. The residual error of the calibration (in Eq. 5) for the proposed method is $0.14 \mathrm{~mm}$ while the residual error for the conventional wall calibration technique is $0.91 \mathrm{~mm}$. Standard deviation of the calibration parameters for 20 trials for both methods have been summarized Table 1.

Table 1. Standard deviation of the calibration parameters for 20 trials, each with 40 ultrasound images of the phantom.

\begin{tabular}{|ccccccc|}
\hline & $t_{x}(\mathrm{~mm})$ & $t_{y}(\mathrm{~mm})$ & $t_{z}(\mathrm{~mm})$ & $r_{x}\left(^{\circ}\right)$ & $r_{y}\left({ }^{\circ}\right)$ & $r_{z}\left({ }^{\circ}\right)$ \\
$\begin{array}{c}\text { Conventional single wall technique } \\
\text { Closed-form differential single wall } \\
\text { technique }\end{array}$ & 1.3949 & 2.1482 & 1.0877 & 1.1510 & 2.2580 & 0.6764 \\
\hline
\end{tabular}




\section{CONCLUSION}

For the first time, a novel closed-form solution for the conventional single wall technique is proposed. Another novel idea is using differential measurements to perform calibration. By performing calculations on differential measurements (line slope) using the closed-form solution more accurate results are obtained compared to the conventional single wall technique ${ }^{5}$.

The closed-form solution can give insight for understanding error propagation and for finding the optimal set of transducer poses. Also the closed-form solution provides fast convergence to the solution and is useful to avoid wrong sub-optimal solutions.

Accuracy can be improved further by using RF data instead of B-mode images especially since it is easy to find line slopes by cross correlation of any two RF echo pulses. Accurate measurement is possible by using new methods of ultrasound motion tracking ${ }^{8}$. This is the future goal of this work.

\section{ACKNOWLEGMENTS}

This work is supported by the Natural Sciences and Engineering Research Council of Canada (NSERC) and Canadian Institutes of Health Research (CIHR).

\section{REFERENCES}

[1] Hsu, P., Prager, R., Gee, A., and Treece, G., "Freehand 3D Ultrasound Calibration: A Review," Advanced Imaging in Biology and Medicine, 47-84 (2009).

[2] Mercier, L., Langø, T., Lindseth, F., and Collins, D.L., "A review of calibration techniques for freehand 3-D ultrasound systems," Ultrasound in Medicine \& Biology 31(4), 449-471 (2005).

[3] Viswanathan, A., "Immediate Ultrasound Calibration from Three Poses and Minimal Image Processing," Proceedings in Lecture Notes in Computer Science Medical Image Computing and Computer-Assisted Intervention (MICCAI) 3217, 446-454 (2004).

[4] Chen, T.K., Thurston, A.D., Ellis, R.E., and Abolmaesumi, P., "A Real-Time Freehand Ultrasound Calibration System with Automatic Accuracy Feedback and Control," Ultrasound in Medicine \& Biology 35(1), 79-93 (2009).

[5] Prager, R., "Rapid calibration for 3-D freehand ultrasound," Ultrasound in Medicine \& Biology 24(6), 855-869 (1998).

[6] Boctor, E.M., Iordachita, I., Choti, M.A., Hager, G., and Fichtinger, G., "Bootstrapped ultrasound calibration," Studies in Health Technology and Informatics 119, 61-66 (2006).

[7] Afsham, N., Chan, K., Pan, L., Tang, S., and Rohling, R.N., "Alignment and calibration of high frequency ultrasound (HFUS) and optical coherence tomography (OCT) 1D transducers using a dual wedge-tri step phantom," Proceedings of SPIE 7964(1), 796428-796428-8 (2011).

[8] Zahiri-Azar, R., and Salcudean, S.E., "Motion Estimation in Ultrasound Images Using Time Domain Cross Correlation With Prior Estimates," IEEE Transactions on Biomedical Engineering 53(10), 1990-2000 (2006). 